\title{
TITEL
}

Prof. Dr. Dr. Michael Bock

\section{Die missliche Lage der kriminalpolitischen Kriminologie. Eine kritische Stellungnahme zum „Freiburger Memorandum“1}

Vorbemerkung: Wie in zablreichen davor geschriebenen lässt sich auch in diesem Text eine Zwangslage nicht auflösen: So oder so bin ich der Hauptautor der früher in Tübingen heimischen und nach Mainz ausgewanderten Angewandten Kriminologie ${ }^{2}$. Wenn ich nicht schweigen will - und als Professor, der die Kriminologie in Lehre und Forschung vertritt, kann ich das nicht - bin ich mit allem, was ich kritisch gegen den mainstream in diesem Fach vorbringe, dem Verdacht ausgesetzt, pro domo zu sprechen. Da sich dies ohnebin nicht vermeiden lässt, sei es hier gleich zu Anfang offen ausgesprochen. Gleichwobl hoffe ich auf Leser, die sich unabhängig davon auf die hier angesprochenen Fragen einlassen.

Nun haben wir also das „Freiburger Memorandum“. Was daran zunächst einmal auffällt, ist die durchgehende Rhetorik der Rechtfertigung: Was für die Zukunft nottut, ist vor allem das, was man selbst in der Vergangenheit gemacht hat. Und: Die Misere ist in keiner Weise selbst verschuldet. Es sind die juristischen Fakultäten und dort insbesondere die unbelehrbaren Strafrechtler, die sozialwissenschaftlichen Fakultäten, die unberechtigte Unterbewertung der deutschen Kriminologie im internationalen Vergleich, natürlich insgesamt die Unterfinanzierung des Faches, wo es doch eine so große gesellschaftliche Bedeutung hat und vor allem haben könnte. Im Ergebnis leidet die Kriminologie „an einer strukturbedingten Auszehrung" (S. 15). Ähnlich klingt es auch in unterschiedlicher Weise im Editorial sowie in den Äußerungen der Herausgeber.

Solche Argumentationsmuster sind auch aus anderen Kontexten gut bekannt. Auch Heime, Vollzugsanstalten, Kliniken oder Ministerien haben immer alles richtig gemacht (aus kriminologischer Sicht ist dies die weit verbreitete „paradoxe Anpassungserwartung"). Niemand aus dem Berliner Ministerium wird sagen, man habe mit dem Bachelor

1 Albrecht/Quensel/Sessar NK 1/2013, 10-15. Vgl. zu diesem Thema auch das Editorial NK 1/2013, 8f., einen kommentierten Bericht über die diesem Memorandum vorausgehende Tagung in Freiburg von Drenkhahn NK 1/2013, 16-19 sowie eine Befragung der Herausgeber der NK NK 1/2013, 26-47. Seitenzahlen im Text beziehen sich alle auf dieses Heft der NK.

2 Bock 2007, 133-158. 
die deutschen Universitäten ruiniert. So etwas kommt immer heraus, wenn man diejenigen nach den Gründen einer Misere fragt, die sie angerichtet haben. Weder was die angeblichen Leistungen in der Vergangenheit betrifft, noch was die Gründe sind, wieso man auch in Zukunft die Kriminologie braucht oder auf sie hören sollte, vernimmt man irgendein wissenschaftliches Sachargument. Stattdessen herrscht der institutionelle Sprachstil von Verwaltern von Wissenschaft vor, eine an Verbandsfunktionäre erinnernde Rhetorik von Studiengängen und Forschungsorganisation, Modulen und Institutionen, Berufschancen und Hochschulkarrieren. Weder für die Lehre noch für die Forschung erfährt man mehr als die Themen und Felder, aber gerade - außer der leerformelhaften Bezeichnung kriminologisch - wenig über die Inhalte, die gelehrt werden sollen und über die man sich durch Forschung Klarheit verschaffen will. Was und zu welchem Ende die entsprechenden Studierenden denn lernen sollen und warum es für das Leben in diesem Gemeinwesen gut sein könnte, Lehrstühle und kriminologische Forschungseinrichtungen zu finanzieren, bleibt im Dunkeln. In der Ansage, dass eine "Chance vertan“ (S. 15) werden könnte, steckt insofern nicht viel mehr als eine Prätention (oder subtile Drohung), weil man als Leser gar nicht erkennen kann, was denn eigentlich von solchem Wert sein soll, dass es mit negativen Folgen vertan werden könnte.

Zum Verständnis des im Folgenden eingenommenen Standpunktes der Angewandten Kriminologie zunächst eine kleine Übersicht:

\section{Praxisorientierung kriminologischer Richtungen}

\begin{tabular}{|l|l|l|l|}
\hline & Praxisfeld & Ziel & Adressat \\
\hline $\begin{array}{l}\text { Kritische } \\
\text { Kriminologie }\end{array}$ & Diskurs & $\begin{array}{l}\text { Delegitimierung } \\
\text { von Kontrolle und Kriminalisierung }\end{array}$ & Intellektuelle \\
\hline $\begin{array}{l}\text { Kriminalpolitsche } \\
\text { Kriminologie }\end{array}$ & $\begin{array}{l}\text { Planung der } \\
\text { Strafrechts- } \\
\text { pflege }\end{array}$ & $\begin{array}{l}\text { Optimierung strafrechtlicher und } \\
\text { gesellschaftlicher Kriminalprävention }\end{array}$ & Eliten \\
\hline $\begin{array}{l}\text { Angewandte } \\
\text { Kriminologie }\end{array}$ & $\begin{array}{l}\text { Arbeit in der } \\
\text { Strafrechts- } \\
\text { pflege }\end{array}$ & $\begin{array}{l}\text { Spezialpräventive } \\
\text { Interventionsplanung im Einzelfall }\end{array}$ & Praktiker \\
\hline
\end{tabular}

Quelle: Bock, Standortbestimmung der Angewandten Kriminologie, in: Liebl (Hrsg.), Kriminologie im 21. Jahrhundert, 2007, S. 27-41, erweitert.

Seit Franz von Liszt die spezialpräventive Imprägnierung unseres Strafrechts initiiert hat, ist der strafrechtliche Normenbestand förmlich durchtränkt worden von entsprechenden Vorschriften, die bis heute in Geltung sind. Notabene - das muss man heute nicht nur Kriminologen, sondern auch den Obergerichten sagen - hat der demokratische Souverän dies so gewollt. Die Strafjustiz allerdings schert sich darum wenig. Mit dem nassforschen Anspruch „wir sind die Praxis“ findet täglich eine massenhafte, an Rechtsbeugung grenzende rechtswidrige Entscheidungsfindung statt, weil die Justiz die Grundsätze der Individualität (präventive Maßnahmen ergehen höchstpersönlich), der Aktualität (die Gefahr muss gegenwärtig sein), der Vollständigkeit der Sachverhaltsermittlung (diese ist bei Auslegung unbestimmten Rechtsbegriffen und bei der Ermessensausübung rechtlich ge- 
fordert) und der konkreten Interventionsplanung (das ist bei Zweckprogrammen rechtlich gefordert) konsequent missachtet. ${ }^{3}$ Und dies noch nicht einmal mit schlechtem Gewissen. Die meisten Juristen wissen nicht, was sie tun, sondern folgen den Routinen „der Praxis" und bekommen noch die Absolution des Kriminologen, der weitergehende Anforderungen als „Luxus“4 bezeichnet.

Es wird also täglich und vor aller Augen (d. h. nicht in unzugänglichen Terrorcamps, Regierungen von Schurkenstaaten oder Chefetagen von Banken) überflüssiges und schweres Leid für Täter und Opfer produziert, das der Gesetzgeber verhindern wollte und für das er sich eine Wissenschaft wie die Kriminologie geleistet hat, wohl wissend, dass die Strafrechtsdogmatik allein den Juristen dafür nicht instand setzt. ${ }^{5}$ Das ganze Reden von der Kriminologie als einem "Annex“ oder einer „Hilfswissenschaft" des Strafrechts würde sofort verstummen, wenn man diesen einfachen und letztlich konsentierten Befund ernst nehmen würde, durch den sich die Verhältnisse in gewisser Hinsicht geradezu umkehren: Das Jugendstrafrecht und das Strafvollzugsrecht, letztlich das gesamte Sanktionenrecht, soweit es nicht vom Vergeltungsgedanken bestimmt ist, sind bei Lichte besehen „akzessorisch“ zur Kriminologie, und zwar ist dies fatalerweise eine Akzessorietät außerhalb der Kompetenzen des Einheitsjuristen, der wohl die Fremdheit einer Sache oder die Gültigkeit einer Betriebserlaubnis beurteilen kann, aber nicht das Vorliegen schädlicher Neigungen, die Gefahr zukünftiger Straftaten oder die Behandlungserfordernisse in einem Vollzugs- oder Förderplan. Ohne Kriminologie keine Strafrechtspflege, die den Anforderungen des geltenden Rechts genügt. Nicht umsonst beginnt denn auch für Franz von Liszt die wissenschaftliche Aufgabe der Strafrechtswissenschaft erst nach der Dogmatik.

Von alldem schweigt das Memorandum, obwohl es sonst alles akribisch notiert, was als Aufweis der Notwendigkeit von möglichst viel Kriminologie in Betracht kommen könnte. Das Editorial erinnert in wenigen Zeilen an dieses Praxisfeld, aber leider auch schon verengt auf „Kriminalprognose“ und den „Markt“ der Gutachten (S. 9). Und auf die Frage nach dem „Täter" in der Herausgeber-Befragung kommen in verschiedener Mischung Platzhalter wie Gewalt- und Sexualtäter, Intensivtäter, Täter mit Migrationshintergrund usw., die sich alle darin gleichen, dass ein statistisches Wissen über Merkmale, Verhaltensweisen, generelle kriminelle Gefährdung usw. gemeint ist, aber nicht die Bedeutung der Frage nach dem individuellen Täter für die tägliche Entscheidungspraxis der Gerichte (so wie etwa die $\$ \$ 46,56$ und 57 StGB danach fragen).

Warum eigentlich? Nicht nur die juristischen Fakultäten, sondern auch die Justizverwaltungen, die das Referendariat organisieren, wären jedenfalls mit einer Botschaft konfrontiert (ich dachte immer, ein Memorandum muss eine Botschaft haben und nicht nur Ansprüche formulieren), die sie zwar vermutlich auch mit Macht und/oder Alternativlosigkeitsrhetorik abwehren würden (Argumente der sogenannten „Praxistauglichkeit“

3 Im Einzelnen ausgearbeitet findet sich dies in der 4. Aufl. meines Lehrbuchs (Bock 2013), in Ansätzen auch schon in Bock 2011b, 459-473.

4 Angesicht der Notwendigkeit einer möglichst zeitnahen Bearbeitung könne sich die Praxis „den Luxus des wissenschaftlich-theoretischen Blicks" nicht leisten (Meier 2010, 501).

5 Bock 2010, 139-167. 
oder der „Pensen“ oder überhaupt der „Finanzierbarkeit“ oder „Darstellbarkeit“), aber sie müssten sich wenigstens mit dem Vorhalt auseinandersetzen, willentlich und wissentlich massenhaft Unrecht zu produzieren.

Diesen Trumpf gibt das Memorandum bzw. das Fach, das sich diese Visitenkarte gibt, ohne Not auf und darf sich dann natürlich nicht wundern, wenn dezimiert wird, was sich selbst nicht ernst nimmt oder nicht sieht, wo seine Stärken sind. Und dies meine ich eben nicht nur taktisch und strategisch im Hinblick auf Berufungschancen oder Forschungsgelder (das kann dann vielleicht am Ende auch daraus folgen), sondern mit Blick auf die Frage, welches Gemeinwesen wir sein wollen und ob es zum Bild dieses Gemeinwesens gehört, massenhaft unnötiges Leid zu produzieren.

Um dies wenigstens an einem Beispiel zu verdeutlichen sei kurz auf unser sogenanntes Wiesbadener Verlaufsprojekt eingegangen ${ }^{6}$. Die Besonderheit dieser Untersuchung besteht darin, dass ihre Methodik einen inhaltlichen Maßstab für die Angemessenheit der getroffenen rechtlichen Entscheidungen liefert, in diesem Fall der Verhängung einer Jugendstrafe ohne Bewährung. Anders als in der Sanktionsforschung üblich, konnte nämlich der input der jugendstrafrechtlichen Entscheidungen sehr wohl kontrolliert werden, und zwar nicht durch eine einfache Checkliste von Risikofaktoren, sondern mit vollständigen biographischen Einzelfallanalysen auf der Grundlage der Angewandten Kriminologie ${ }^{7}$.

Schon gleich das Ergebnis unserer Zugangs- oder Basisdiagnostik war überraschend und irritierend. Die Probanden sind alles andere als eine einigermaßen homogene Gruppe, wie man dies erwarten sollte, wenn man an die jugendstrafrechtlichen Voraussetzungen der Verhängung, Bemessung und Vollstreckung einer unbedingten Jugendstrafe denkt. Denn bei 10 von 40 Probanden lag eine biographische Nähe zur Kriminalität im Rabmen der Persönlichkeitsreifung vor und dies bedeutet eine klare Kontraindikation für das Vorliegen der Voraussetzungen einer Jugendstrafe. Bei der Erwartung einer vorübergehenden kriminellen Episode mit Delikten von geringer Zielstrebigkeit, wie es diese Verlaufsform impliziert, liegen nach herrschender Meinung und ständiger Rechtsprechung weder schädliche Neigungen noch Schwere der Schuld im Sinne von $\mathbb{} 17$ Abs. 2 JGG vor. Kommt man ausnahmsweise mit Rücksicht auf das sogenannte Gerechtigkeitsgefübl der Bevölkerung aufgrund der objektiven Schwere des Tatunrechts (die notabene eigentlich unerheblich ist) zur Verhängung einer Jugendstrafe wegen Schwere der Schuld, so führt unweigerlich die Bemessung dieser, die erzieherisch erfolgen muss und bei der die Strafrahmen des allgemeinen Strafrechts ausdrücklich nicht gelten ( $\$ 18$ Abs. 2 JGG), zu einer Jugendstrafe am unteren Ende der Möglichkeiten (Mindeststrafe 6 Monate). Dies aus dem einfachen Grund, dass bei einer Kriminalität im Rabmen der Persönlichkeitsreifung der Jugendstrafvollzug im Vergleich mit ambulanten Maßnahmen keine positiven, sondern allenfalls negative erzieherische Effekte hat und von einem Übel

6 Bock 2011a.

7 Für die Einzelheiten der dabei verwendeten Methode der idealtypisch-vergleichenden Einzelfallanalyse (MIVEA) und ihre Begrifflichkeit wird noch einmal auf mein Lehrbuch (wie Fn. 3) verwiesen.

NK 25. Jg. $4 / 2013$ 
sollte es so wenig wie möglich geben. Spätestens aber bei der Frage der Vollstreckung hätte Schluss sein müssen, denn bei einer Kriminalität im Rabmen der Persönlichkeitsreifung liegt genau die positive Prognose vor, die das Gesetz (\$21 JGG) fordert. 10 von 40 Probanden hätten also nie in den Vollzug kommen dürfen und weitere 9 bei richtiger Auslegung des Gesetzes auch nicht, denn bei diesen 9 Probanden lagen Zwischenformen zwischen Kontinuierlicher Hinentwicklung zur Kriminalität und Kriminalität im Rabmen der Persönlichkeitsreifung vor und für diese Konstellation sieht das JGG die Aussetzung der Verhängung einer Jugendstrafe zur Bewährung vor (\$27 JGG).

Man sieht an diesem Ergebnis übrigens die Vergeblichkeit des Versuchs, die Wirksamkeit des Jugendstrafvollzugs mit Rückfallzahlen messen zu wollen. Wenn schon ungefähr die Hälfte gar nicht in den Jugendstrafvollzug hätte kommen dürfen, kann man auch nicht seine Wirkung prüfen.

Was sich schon in diesen ersten Ergebnissen abzeichnet, ist eine erschütternde Überforderung der Gerichte - immerhin meist Jugendkammern am Landgericht - mit der Auslegung der zentralen Begriffe des JGG. Die Ausführungen in den Urteilen sind überwiegend unsäglich ${ }^{8}$, die zentrale Vorschrift über die Bemessung der Jugendstrafe wird in keinem einzigen der Urteile angemessen ausgelegt. Erstaunlich ist nur, warum immer über die Jugendkriminalität Klage geführt wird und Forderungen nach Strafverschärfungen (etwa: Warnschussarrest; früher war die Richtung der Forderungen eine andere) erhoben werden, aber niemand fragt, ob die Verfahrensbeteiligten für ihre Aufgabe überhaupt ausgebildet sind.

Nun ist dies freilich noch nicht die ganze Geschichte. Seit sich die Kritische Kriminologie mit großer gesinnungsethischer Emphase und mit Rücksicht auf ihre wissenschaftliche „Reinheit“ in den Norden abgesetzt hat und seit die Südwestdeutsche Kriminologie sich nur noch mit kriminalpolitisch relevanten Themen beschäftigt (das war nicht immer so, und in den 70er Jahren war vieles möglich), wird von ihnen gebetsmühlenartig behauptet, dass die Kriminologie die vorgenannte Aufgabe der Begrenzung von Leid durch spezialpräventive Expertise im Einzelfall nicht übernehmen dürfe und/oder nicht könne, wobei das nicht Dürfen bei der Kritischen Kriminologie sozusagen grundständig (gesinnungsethisch) begründet wird und bei der Kriminalpolitischen Kriminologie erst aus dem nicht Können folgt. Den etablierten Kolleginnen und Kollegen erspare ich eine weitere Reprise der Auseinandersetzung, die ich mit diesen Positionen seit Jahr und Tag führe", und den wissenschaftlichen Nachwuchs verweise ich auf meine Lehrstuhlseite (kommentierte Bibliographie zur Einzelfallkriminologie) und die 4. Auflage meines Lehrbuchs. Deshalb hierzu nur ein kleiner wissenschaftstheoretischer Syllogismus. Obersatz: Mit nomothetischer Forschungslogik gewonnene Resultate besagen für die Einzelfallentscheidungen der Praxis nichts. Untersatz: Die Kriminologie des Memorandums forscht nomothetisch. Conclusio: Ihre Resultate besagen für die Einzelfallentscheidungen der Strafrechtspflege nichts.

8 Eine Blütenlese findet sich bei Bock 2011b (wie Fn. 2).

9 Vgl. außer den in Fn. 10 genannten Arbeiten schon früher Bock 1999b, 285-303 sowie ders. 1999a, 625-638. 
Es ist diese, nicht schicksalhaft oder „strukturell“ bedingte, sondern selbst gewäblte wissenschaftstheoretische Ausrichtung, durch die sich die Kriminologie des Memorandums selbst ihre Kompetenz für die Strafrechtspraxis beschnitten hat. Dass man sich insoweit gegenüber der Psychiatrie nicht „behaupten konnte“ (Editorial, S. 9), ist im Ergebnis richtig, verkennt aber den Umstand, dass die Kriminologie des Memorandums sich von sich aus dieses Praxisfeldes nicht nur entledigt, sondern auch noch diejenigen (eigentlich wäre hier der Singular angebracht) bekämpft hat, die versucht haben, dort das Vorrecht des spezifisch kriminologischen Blicks auf das Verbrechen gegenüber den Bezugswissenschaften zu verteidigen. ${ }^{10}$ Bis heute gibt es dafür keinerlei Unterstützung, sondern bestenfalls Schweigen.

Es geht dabei auch gar nicht in erster Linie um die Erstellung von Gutachten, sondern um die massenhafte tägliche Entscheidungspraxis, in der die Psychiatrie zwar noch nicht so weit durchgedrungen ist, dass Juristen und Sozialarbeiter flächendeckend psychiatrisch statt kriminologisch geschult werden, aber es sind doch deutliche Ansätze in diese Richtung zu verzeichnen, etwa in der Bewährungshilfe, wo eine kriminologo-phobe und psychiatro-phile Strafrechtspflege das Sagen hat und Sozialarbeiter etwa auf die insoweit sach- und fachfremden Dittmann-Kriterien vergattert. Nein, diese ganze Misere ist nicht das Resultat opaker Strukturen, sondern von (aus heutiger Sicht unnötig verletzenden) Richtungskämpfen der 60er und 70er Jahre, in denen sich unser Fach im Wesentlichen seine heutige Gestalt gegeben hat. ${ }^{11}$ Wenn darauf hier mit Nachdruck bestanden wird, so geht es dabei gar nicht um Nachkarten oder die Begleichung alter Rechnungen (fraglos gab es auch persönliche Kränkungen), sondern in dem Sinn um die Zukunft der Kriminologie, als die Erinnerung an die im Wortsinn historische Genese der Gestalt der heutigen Kriminologie diese als eine Gemachte und damit auch als eine Veränderbare zeigt.

Aufgrund der damaligen Weichenstellungen gilt nun freilich, dass zwar kriminologische Themen auf der Straße liegen (Drenkhabn im Heft S. 18), aber bezüglich dieses Themas wäre (im Sinne eines Müller-Dietz'schen Aphorismus zum Gelde) zu präzisieren, es liegt vor allem deshalb auf der Straße, weil es zum Fenster hinausgeworfen wurde. Und für diejenigen, die sich noch damit befassen gilt, (ebenfalls mit Müller-Dietz): wer mit dem Rücken zur Wand steht, kann nicht umfallen.

Der Bezeichnung der Themen im Memorandum nach (Längsschnittuntersuchungen, Intensivtäter, Gewalt- und Sexualdelikte, Jugendkriminalität, Wirtschaftskriminalität,

10 Es geht hier in erster Linie um das Verhältnis zur forensischen Psychiatrie und Psychologie, vgl. dazu folgende meiner Arbeiten: Bock, StV 5/2007, 269-275; ders. Zeitschrift für die gesamte Strafrechtswissenschaft 2009, 450-463; ders. 2012 609-620. Von ähnlicher praktischer Bedeutung ist aber auch das ganze Berufsfeld der sozialen Dienste in der Justiz, vgl. hierzu Bock 2009, 101-133. Ganz frisch erschienen ist Brockmann/Bock FPPK 2013, 133-140 sowie 193-201.

11 Vgl. etwa Müller-Dietz (Hrsg.) 1994 sowie Kaiser 2007, 11 ff., wo sehr dezent von „zwischenzeitlichen Friktionen, Turbulenzen und Veränderungen“ (S.13) die Rede ist. Die Jüngeren mögen im Übrigen über diese Zeit ihre akademischen Lehrer oder sonstige Augenzeugen befragen, denn es werden hierzu - wie wir es alle gelernt haben - nur Versionen berichtet (vgl. etwa auch die Beiträge in Heft 2 des KrimJ von 2009), die aber alle jedenfalls darin übereinstimmen, dass damals heftig gekämpft und gestritten wurde und man nicht nur Zuschauer eines gleichsam naturwüchsigen Entwicklungsprozesses war.

NK 25. Jg. 4/2013 
Kriminalität von Migranten usw. usf.) könnte es noch so scheinen, als ob die Einzelfallentscheidungen der Strafrechtspflege noch gemeint seien, aber wenn man auf die Art des Wissens blickt, kommt es überdeutlich an den Tag, dass dies nicht der Fall ist. Dass z. B. die Jugendkriminalität meistens episodenhaft und die Fluchtgefahr bei Migranten bäufig überschätzt wird, oder dass bei der Verhängung von Jugendarrest oft weitere Straftaten folgen, ist für die Arbeit am und mit dem konkreten Fall irrelevant, ja, man könnte sogar auf die Idee kommen, diese Art von Wissen erzeuge eine rechtlich problematische Voreingenommenheit für diesen jetzt $\mathrm{zu}$ entscheidenden Fall. Die nur noch kriminalpolitische Relevanz war ja gerade ein wesentliches Element des Reinheitsgebotes der Kriminalpolitischen Kriminologie und sollte ihre wissenschaftliche Unabhängigkeit verbürgen, aber diesen Umstand vernebeln das Memorandum und die Antworten der Befragung konsequent.

Anders ist dies nur bei der Angewandten Kriminologie ${ }^{12}$, aber die kommt im Memorandum und in der (bisherigen) Herausgeber-Befragung nicht vor, weder mit ihren wissenschaftlichen Methoden und Leistungen noch mit ihrer Unentbehrlichkeit für die tägliche Praxis der Strafrechtspflege im Einzelfall, aus der und nur aus der letztlich die Notwendigkeit und Berechtigung folgt, nicht nur Intellektuelle und Eliten in Ministerien und sonstigen Leitungsfunktionen kriminologisch zu informieren, sondern die große Zahl der Juristen, Sozialarbeiter, Polizisten und sonstigen Personen, deren Beruf über Jahre und Jahrzehnte nicht kritische Reflexion und/oder politische Planung ist, sondern Arbeit mit Einzelfällen und also mit Menschen.

Jeder in diesem Fach Tätige muss sich sein eigenes Bild machen, denn eine herrschende Meinung gibt es in einer Erfahrungswissenschaft nicht, jedenfalls nicht in einer, die noch eine sein will. Insofern haben auch die Meinungen und Positionen des Memorandums nicht deshalb recht, weil sich dort die herrschenden Kriminologen (sogar „über 60“ von ihnen) geäußert haben. Wer zu dem Ergebnis kommt, die Kriminologie würde ihre Selbständigkeit und/oder ihre Wissenschaftlichkeit verlieren, wenn sie sich der oben angesprochenen großen Aufgabe spezialpräventiver Interventionsplanung im Einzelfall widmen würde (das Gesetz repräsentiert insoweit nichts weniger als die abendländische

12 Es ist an dieser Stelle nicht möglich, diese kriminologische Position auch nur halbwegs angemessen zu präsentieren, weshalb der interessierte Leser abermals auf das in Fn. 3 genannte Lehrbuch sowie auf eine umfangreiche kommentierte Bibliographie verwiesen werden muss (http://www.jura.uni-mainz.de/bock/289.php). Die Angewandte Kriminologie ist im Fach vor allem als etwas unter allen Umständen zu Kritisierendes bekannt (es wäre interessant gewesen, die Herausgeber zu fragen, ob ihnen diese Position überhaupt aus eigener Lektüre vertraut ist), wodurch weitgehend in Vergessenheit geraten ist, dass sie zwar einen besonderen Zugang zum Straftäter als Individuum und damit zur Praxis der Strafrechtspflege eröffnet, dass sie aber in vieler Hinsicht und bis in die Einzelheiten hinein mit den sonstigen relevanten kriminologischen Erkenntnisbemühungen (etwa der Entwicklungskriminologie) konvergiert oder sie vorweggenommen hat (wie etwa die bekannten Verlaufstypen von Moffitt) bzw. deren Leistungen auch dort anerkennt, wo sie aus anderen wissenschaftstheoretischen und/oder fachspezifischen Kontexten stammen. Tatsächlich hält sie mit der Erfassung des Täters in seinen sozialen Bezügen eine in Lehre, Weiterbildung, Forschung und gutachterlicher Praxis über viele Jahre erprobte und genau die Leistung vor, von der oben die Rede war und die die Strafrechtspflege um den Preis der massenhaften Produktion von Unrecht ignoriert. 
Tradition), wer also zu dem Schluss des nicht Dürfens und/oder nicht Könnens kommt, muss dann auch zu dem weiteren Schluss kommen, dass die Kriminologie für die Ausbildung von Praktikern gleich welchen Faches oder Tätigkeitsfeldes irrelevant ist. Der allseits registrierte Schwund wäre demnach völlig berechtigt, und in der Juristenausbildung könnte weiterhin (wie es ohnehin schon immer war) spätestens im Referendariat Schluss damit sein, wo es dann endlich um das in den Augen der Justiz „Eigentliche“ geht, und wo schon jetzt von vielen Ausbildern die rechtswidrigen und vor unverhohlener Verachtung für die Kriminologie (zunehmend übrigens auch für Strafrechtsdogmatik) nur so strotzenden Routinen der sogenannten „Praxis“ eingebläut werden.

Bleiben die Leistungen (und Verheißungen) der Kriminologie auf ihrem selbst gewählten Gebiet: der kriminalpolitischen Rationalisierung und Effektivierung der Strafrechtspflege, ggf. ausgreifend in die Makrokriminalität und damit auch in historische, ethnologische, politikwissenschaftliche usw. Studien, selbstredend interdisziplinär und international, organisatorisch vernetzt, englischsprachig und diversity-konform. Das wäre bei Franz von Liszt die kriminalpolitische Aufgabe der Gesamten Strafrechtswissenschaft.

Dass diese Aufgabe auch eine berechtigte und wichtige ist, ist von mir nie ${ }^{13}$ und soll auch hier in keiner Weise in Frage gestellt werden. ${ }^{14}$ Insoweit zahlt die Angewandte Kriminologie nicht mit gleicher Münze heim. Vorbehalte ergeben sich nur wieder bezüglich der Art und Weise, wie das Memorandum sich hierzu äußert, nämlich so, als ob die Kriminologie diese Aufgabe im Wesentlichen gelöst habe und - sofern nicht durch strukturelle Auszehrung Chancen vertan werden - auch in Zukunft fortlaufend besser und umfassender lösen werde (etwa S. 10 unten und S. 13 unten). Als ob ein gut gefüllter Fundus bereit stünde, bei dem die Entscheidungsträger nur zugreifen müssten und man auch den gesellschaftspolitischen Diskurs über Kriminalität und Kriminalitätskontrolle maßgeblich bestimmen könne. Es wird hier ein gewissermaßen automatischer „Erfolg“ von Forschung unterstellt, den es weder in der Vergangenheit gab noch in Zukunft geben wird, d. h. es wird in einer absichtlichen oder unabsichtlichen Realitätsverkennung Anspruch und Wirklichkeit der Forschung verwechselt. Tatsächlich harrt der alte positivistische (rationalistische) Traum weiter seiner Erfüllung, denn die Gegenstände und Themen sind komplex und spröde gegenüber den eingesetzten (und als allein „wissenschaftlich" behaupteten) Methoden, denen sie sich angeblich reibungslos fügen. Mit der Folge, dass - und das spricht gar nicht gegen Ernst und Qualität der Versuche - bei den Resultaten der Forschung neben einigen wenigen Leuchtturmprojekten eine große Masse an sterbenslangweiligem deskriptivem Material produziert und respektable Datenfriedhöfe angelegt worden sind, denen man selbst mit dem besten Willen keinen und schon gar keinen eindeutigen kriminalpolitischen Sinn abgewinnen kann.

13 Vgl. etwa Bock 2007, wie Fn. 2.

14 Auch hier ist eine angemessene Dokumentation allerdings illusorisch, weshalb neben den Periodischen Sicherheitsberichten der Bundesregierung exemplarisch verwiesen wird auf Höfer/ Spiess (Hrsg.) 2007.

NK 25. Jg. $4 / 2013$ 
Die „Daten“, die man zum Rechnen nimmt (immer noch herrscht die Ansicht vor, durch Zahlen und Rechnen erweise sich die Kriminologie als empirische Wissenschaft), sind gar keine „Daten“, denn sie sind nicht „gegeben“, sondern „gemacht“, etwa „gedealt" von den Institutionen der Strafrechtspflege, die damit ihre (Un-)Tätigkeit dokumentieren. Auch „Daten“ aus Befragungen sind keine „Daten“, sondern Reaktionen auf die Reize von gestellten und also gemachten Fragen. Die Selektionen, etwa auch durch die notorisch geringen Rücklaufquoten, sind bekannt - man hat sich an die disclaimer gewöhnt und akzeptiert sie, sonst müsste man von „Resultaten“ ganz schweigen - und auch die gewünschten experimentellen designs des evidenzbasierten Goldstandards lassen sich regelmäßig nicht realisieren. Sobald es aber um Themen mit mächtigen Akteuren und großen Gefahren geht (Makrokriminalität, Terrorismus usw. usf.), um die sich die Kriminologie des Memorandums (sozusagen „in brennender Sorge“) besonders kümmern möchte (dadurch erscheint das Vertun einer Chance erst recht fahrlässig), ist es auch mit diesen sogenannten Daten dahin, denn der Feldzugang ist bei diesen Themen bekanntlich eine Machtfrage und daher für Kriminologen schon bei Wirtschaftskriminalität und Korruption schwierig (hier müsste man im Einzelnen differenzieren ${ }^{15}$ ), bei Terrorismus und Staatskriminalität aber ganz verstellt. Kriminologische Texte käuen daher, je schlechter es um den Feldzugang bestellt ist, aus Mangel an eigenen Erkenntnissen überwiegend wieder, was diejenigen finden, denen der Feldzugang noch etwas besser gelingt: dem BKA und seinen internationalen Pendants, den Geheimdiensten und mutigen Journalisten. Selten gibt es einige Fälle, in denen Akten angefallen sind, aber auch sie sagen (wenn man sie überhaupt bekommt) wenig, weil die Selektionen offensichtlich (es sind ja gerade die Fälle, in denen die Strafverfolgung der Gefahr offenbar gewachsen war) und wieder disclaimer fällig sind.

Was sogenannte Hintergründe betrifft, sind Kriminologen - trotz oder wegen ihrer im Memorandum und auch sonst gerühmten juristischen Kenntnisse und Denkweise den Wissenschaftlern aus den einschlägigen Fächern hoffnungslos unterlegen. Kulturund Religionssoziologie, Geschichtswissenschaft, Politikwissenschaft und Ethnologie haben sich mit diesen Fragen schon immer befasst. Die Folge ist, dass in Wort und Bild mit erheblichem moralischem Impetus Spekulationen über die Schäden und die Gefahren der entsprechenden Phänomene vorgetragen werden (jeder hat da sein Steckenpferd; meines wäre der terroristische Angriff auf die Volksgesundheit durch facebook), in der Sache selbst aber werden überwiegend andere Quellen rezipiert, so dass eine gewiss umfangreiche Sekundär- und Tertiärliteratur zu verzeichnen ist, in der aber erneut die disclaimer in den seriösen Arbeiten immer lauter sagen, dass man nicht wirklich viel wisse, schon gar nicht, was zu tun sei (auch dies ist nämlich in der Regel eine Machtfrage), und dann kriminologisch durchaus klug erklären, warum dies so ist. Wird die Kriminologie von Institutionen mit mehr Macht und daher besserem Feldzugang, als sie ihn selbst hat, beauftragt oder beteiligt, so ist sie gebunden und wird gerade dort auf den Status einer in jeder Hinsicht abhängigen Hilfswissenschaft herabgewürdigt, die weder Fragen noch

$15 \mathrm{Vgl}$. etwa die interessante und auch methodisch kreative Wege beschreitenden Untersuchungen von Boemke/Grau/Kißling/Schneider; Denkströme 9/2012, $79 \mathrm{ff.,} 85$. 
Publikationen souverän bestimmt, also genau zu dem (dabei noch schlecht bezahlten) Büttel, den nicht mehr abzugeben sie in den 70er Jahren ausgezogen war. So gibt es insgesamt sehr viel Empörung, sehr viel Forschung, sehr viel Papier und elektronische Texte, aber doch nur wenig Licht und viel Schatten. Von einer fertigen Gebrauchsanweisung für rationale Kriminalpolitik kann beim besten Willen seriöserweise nicht die Rede sein.

Entscheidend ist aber, dass die Kriminalpolitik selbst sich auch um die tragfähigen Resultate der Kriminalpolitischen Kriminologie (wie zum Exempel für die Thesen von Garland) genauso wenig schert wie die Strafjustiz um den gut begründeten Vorhalt ihrer weitgehenden Rechtlosigkeit. Dieser Umstand mag dann wiederum zum Gegenstand durchaus interessanter und anspruchsvoller kritisch-reflexiver Forschung und Ausbildung in Masterstudiengängen werden, von der freilich der Praktiker vor Ort und in den operativen Zwängen seines Alltags erneut wenig profitieren wird. Die Kriminalpolitik benutzt die Kriminalpolitische Kriminologie allenfalls noch als Feigenblatt, wo ihre Resultate auf Linie sind. Sie sind es z. B. immer, wenn eine bisher unterschätzte "Gefahr“ mit besorgter Miene geäußert wird, der sich die Politik annimmt, um "Sicherheit“ herzustellen. Ansonsten ignoriert sie deren Publikationen, aber auch ganz unverhohlen die Sachverständigen in den Anhörungen der Politikberatung, für die dann immerhin noch die mehr oder weniger leckeren Schnittchen bleiben. Es seien hier nur beispielhaft die Themen der häuslichen Gewalt (sogar der Periodische Sicherheitsbericht der Bundesregierung selbst wird in der Praxis des sogenannten Gewaltschutzes ignoriert), des Warnschussarrests und der Sicherungsverwahrung genannt. Hauptsache die Täter werden bestraft, am besten für immer weggeschlossen, und die Opfer entschädigt. Was denn noch?

Fazit: Das Memorandum und auch die meisten Meinungsäußerungen in der bisherigen Herausgeber-Befragung sind Dokumente der Selbstüberschätzung. Daran, was die Kriminologie könnte und wo sie stark wäre, nämlich an der vom Gesetzgeber gewollten täglichen spezialpräventiven Arbeit der Strafrechtspflege zur Begrenzung des durch das Verbrechen erzeugten Leids, beteiligt sie sich mit voller Absicht nicht. Sie verliert damit zunehmend ihre Berechtigung als Lehr- und Ausbildungsstoff für die zahlenmäßig allein relevanten Praktiker der Strafrechtspflege. Was sie möchte und was sie versucht, gelingt ihr allenfalls punktuell und ansatzweise und sie hat starke Konkurrenz.

Ernsthaft gewollt wird kriminologisches Wissen, gleich welcher Art, weder von der Praxis der Strafrechtspflege noch von der Kriminalpolitik. Ein Problem muss man darin nicht sehen, denn so funktioniert unsere Gesellschaft nun einmal. Jede Gesellschaft hält sich ihre Hofnarren und hat nicht nur die Verbrecher und Verbrechen, sondern auch die Strafrechtspflege, die Medien und die Kriminologie, die sie verdient. 


\section{Literatur}

Albrecht/Quensel/Sessar Lage und Zukunft der Kriminologie. Freiburger Memorandum zur Lage der Kriminologie in Deutschland, in: NK 1/2013, 10-15

Albrecht u.a. Lage und Zukunft der Kriminologie - Fragen und Antworten, in: NK $1 / 2013,26-47$

Bock (2009) Angewandte Kriminologie für Sozialarbeiter, in: Sanders/Bock (Hrsg.), Kundenorientierung - Partizipation - Respekt. Neue Ansätze in der Sozialen Arbeit, 101-133

Bock Das Elend der klinischen Kriminalprognose, in: StV 5/2007, 269-275

Bock (2011a) Das Wiesbadener Verlaufsprojekt - Erste Ergebnisse einer kriminologischen Studie und Folgerungen für die jugendstrafrechtliche Praxis (Mainzer Runde 2011)

Bock (2011b) Der vollständig und zutreffend ermittelte Sachverhalt bei der Kriminalprognose; in: Kühl u.a. (Hrsg.): Rom, Recht, Religion - Symposion für Ebert, 459-473

Bock (2012) Die Verwalter der Gefährlichkeit - eine Skizze zum forensischen Gutachterwesen; in: Hilgendorf/Rengier (Hrsg.), Festschrift für Heinz, 609-620

Bock Gibt es noch Platz für die Angewandte Kriminologie in der Gesamten Strafrechtswissenschaft? in: Zeitschrift für die gesamte Strafrechtswissenschaft 2009, 450-463

Bock (1999a) Je weniger desto besser. Wie im Jugendstrafrecht kriminologische Torheiten dogmatisch geadelt wurden, in: Ebert u.a. (Hrsg.), Festschrift für Hanack, 625-638

Bock (2013) Kriminologie. Für Studium und Praxis, 4. Aufl.

Bock (1999b) Schädlich, überflüssig, schmutzig. Die Argumentationen der kriminologischen Verächter der Resozialisierung; in: Feuerhelm u.a. (Hrsg.), Festschrift für Böhm, 285-303

Bock (2010) Über die positive Spezialprävention in den Zeiten des Feindstrafrechts - Die Bedeutung der Angewandten Kriminologie für eine menschliche Kriminalpolitik; in: EDICIJA CRIMEN, Bd. 16; derselbe Beitrag in serbischer Sprache in: CRIMEN JOURNAL FOR CRIMINAL JUSTICE Belgrad 2/2010, 139-167

Bock (2007) Wo ist die Tübinger Kriminologie? Versuch einer Standortbestimmung der Kriminologie in Mainz, in: Höfer/Spiess (Hrsg.), Neuere kriminologische Forschung im Südwesten. Eine Darstellung der Forschungsarbeit aus Anlass des 40. Kolloquiums der südwestdeutschen und benachbarten kriminologischen Institute, 2. Aufl., 133-158

Boemke/Grau/Kißling/Schneider Evidenzbasierte Kriminalprävention in Unternehmen. Wirksamkeit von Compliance-Maßnahmen in der deutschen Wirtschaft - Ein empirisches Forschungsvorhaben; in: Stekeler-Weithofer (Hrsg.), Denkströme 9/2012, $79 \mathrm{ff}$. 
Brockmann/Bock Die Kriminalprognose bei persönlichkeitsgestörten Straftätern. Teil I: Ausgangslage und Potentiale der Angewandten Kriminologie, in: FPPK 2013, 133-140

Höffler/Kaspar/Schneider Editorial zum Titelthema „Lage und Zukunft der Kriminologie“, in: NK 1/2013, 8f.

Kaiser (2007) 40 Jahre gemeinsame Kolloquien Südwestdeutscher und Schweizer kriminologischer Institutionen, in: Höfer/Spiess (Hrsg.), Neuere Kriminologische Forschung im Südwesten. Eine Darstellung der Forschungsarbeit aus Anlass des 40. Kolloquiums der Südwestdeutschen und benachbarten Kriminologischen Institute, 2. Aufl.

Meier (2010) Was nützt, was schadet, was ist ohne Effekt? - Die jugendstrafrechtlichen Sanktionen auf dem Prüfstand, in: Bloy u.a. (Hrsg.), Festschrift für Maiwald, 501ff.

Müller-Dietz (Hrsg.) (1994) Dreißig Jahre südwestdeutsche und schweizerische kriminologische Kolloquien

Kontakt:

Prof. Dr. Dr. Michael Bock

Johannes Gutenberg-Universität

Fachbereich Rechts- und Wirtschaftswissenschaften

Jakob-Welder-Weg 9

55099 Mainz

LSBock@uni-mainz.de 